\title{
Demographic characteristics, aetiology, and assessment of treatment options in leukocytoclastic vasculitis
}

\author{
Alkim Unal Cakiter¹, Ozlem Su Kucuk², Dilek Biyik Ozkaya², Bugce Topukcu², Nahide Onsun² \\ ${ }^{1}$ Medipol Mega University Hospital, Istanbul, Turkey \\ ${ }^{2}$ Department of Dermatovenereology, Medical Faculty, Bezmialem Vakif University, Istanbul, Turkey \\ Adv Dermatol Allergol 2017; XXXIV (2): 104-109 \\ DOI:https://doi.org/10.5114/ada.2017.67071
}

\begin{abstract}
Introduction: Vasculitides are a heterogeneous group of diseases characterized by inflammation of the blood vessel walls. Etiological factors include infections, drugs, connective tissue diseases, and malignancies.

Aim: To examine the demographic characteristics, etiological factors, and treatment options in 75 patients with leukocytoclastic vasculitis.

Material and methods: The study included 75 patients diagnosed with leukocytoclastic vasculitis at our clinic. The patients' medical records were reviewed to determine their age, sex, presence of systemic symptoms, possible etiological factors, laboratory results, types of cutaneous lesions, locations of the lesions, treatment options, and disease course.

Results: There were 43 women and 32 men. Cutaneous lesions affected only the lower limbs in 60 of the 75 patients $(80 \%)$ and usually presented as palpable purpura $(64 \%, n=48)$. Arthralgia $(26.7 \%, n=20)$ was the most frequent extracutaneous symptom. Of the patients with secondary vasculitis, the most common causes were infections and drugs. The mean age of the patients with Henoch-Schönlein purpura was 26.8 years. There was no significant association between age and renal, gastrointestinal, or joint involvement.

Conclusions: The most common form of vasculitis in our study was cutaneous leukocytoclastic vasculitis. In most of the patients it appeared to be idiopathic. Among drugs, antibiotics were the most common etiological factor. In 4 patients, the cutaneous leukocytoclastic vasculitis behaved like the paraneoplastic syndrome.
\end{abstract}

Key words: leukocytoclastic vasculitis, Henoch-Schönlein purpura, etiological factors.

\section{Introduction}

Vasculitides are a heterogeneous group of more than 20 diseases characterised by inflammation and destruction of blood vessels [1, 2]. Inflammation in the vessel wall is the basic pathology and leads to deterioration of vessel integrity and blood flow and thus damage to tissues and systems. Aetiological factors include infections, medications, connective tissue diseases, inflammatory diseases, and malignancies [1-5]. Vasculitides are classified based on their aetiology, diameter of the vessels involved, clinical findings, and affected organ systems.

A new nomenclature for the various forms of systemic vasculitis was published in January 2013. This system of naming vasculitis updated the nomenclature published in 1994 by the first International Chapel Hill Consensus Conference (CHCC) [6]. Skin lesions frequently appear along with vasculitis of small- and medium-di- ameter vessels [7, 8]. Systemic symptoms, such as fever, myalgia, abdominal pain, and arthralgia, are present with the skin lesions. The presence of such findings may indicate a systemic disease. There may be involvement of the renal, gastrointestinal (GIS), musculoskeletal, and the central nervous systems, too.

\section{Aim}

The aim of this study was to determine the clinical signs and symptoms, demographic characteristics, aetiological factors, and treatment options in 75 patients who had been diagnosed with leukocytoclastic vasculitis.

\section{Material and methods}

In total, 75 patients who visited our clinics and were diagnosed clinically and histopathologically with leuko-

Address for correspondence: Dr. Alkım Ünal Çakıter, Department of Dermatovenereology, Medipol Mega University Hospital, No: 1 Dermatoloji Polikliniği Bağcılar, 34200 Istanbul, Turkey, phone: +90 533 5565980, e-mail: alkimunal@hotmail.com Received: 25.06.2015, accepted: 3.03.2016. 
cytoclastic vasculitis (LCV) were enrolled in the study. The age, gender, backgrounds, and family histories of the cases, accompanying systemic diseases, current medications, the season the disease appeared, location and type of lesions found as a result of a dermatological examination, presence of symptoms in lesions, such as pain and pruritus, and the presence of non-cutaneous findings were recorded. Demographic characteristics, histopathological findings, laboratory findings, involved systems, and treatments were reviewed retrospectively. All data related to the patients were obtained retrospectively from patient files.

\section{Results}

Of 75 patients, 43 (57.3\%) were females and 32 $(42.7 \%)$ males; the ratio of females to males was 1.34 . The age of the patients ranged from 4 to 86 years old, and the mean age was 43.5 years.

While palpable purpura was observed in 48 (64\%) patients, and petechial and purpuric macules in 27 (36\%), other accompanying clinical findings were vesicles and bullae in 12 (16\%), ulcers in 7 (9.3\%), subcutaneous nodules in 2 (2.7\%), papules in 5 (6.6\%), and oedema in the hands and feet in 1 (1.3\%) patient. No patient showed livedo reticularis, splinter haemorrhage, pustules, digital necrosis, or urticarial lesions. Lesions were located only in the lower extremities in $80 \%(n=60)$ of the patients, widely in $8(10.6 \%)$, in the trunk and lower extremities in 4 (5.3\%), lower and upper extremities in 2 (2.6\%), and only in the trunk in $1(1.3 \%)$ patient. When the patients were assessed in terms of aetiological factors, infection was seen in 13 (17.3\%) patients, medication usage in 4 (5.3\%), a combination of infection and medication usage in 5 (6.6\%), a combination of malignancy and rheumatoid arthritis in 1 (1.3\%), rheumatoid arthritis in 1 (1.3\%), Sjögren's syndrome in 1 (1.3\%), a combination of Sjögren's syndrome and infection in 1 (1.3\%), ulcerative colitis in 1 (1.3\%), Crohn's disease in 1 (1.3\%), and cryoglobulinemic vasculitis in 1 (1.3\%), and 20 (20.6\%) patients were assessed as idiopathic.

Henoch-Schönlein purpura (HSP) ranked the first, at a frequency of $32 \%(n=24)$. In $15(62.5 \%)$ patients with HSP, there was an association with an infection occurring within the last 2 weeks.

In cases in which a suspicious medication was used shortly before the onset of the rash, there was no other apparent reason contributing to the aetiology, and the lesions regressed upon discontinuation of the medication, thus the vasculitis was deemed to be associated with the use of the medication. While use of the medication alone was found in 4 patients, combinations of the medication and infection were seen in 5 patients. These medications included antibiotics $(n=4)$, non-steroidal anti-inflammatory drugs (NSAIDs; $n=2$ ), calcium channel blockers $(n=1)$, propylthiouracil $(n=1), \beta$-blocker $(n=1)$, angiotensin converting enzyme (ACE) inhibitors $(n=1)$, lamotrigine $(n=1)$, and ursodeoxycholic acid $(n=1)$.

There was a previously diagnosed malignancy in 3 of 4 patients with detected malignancies. These were breast cancer $(n=1)$, malignant melanoma $(n=1)$, and Kaposi's sarcoma $(n=1)$. In 1 patient, non-Hodgkin's lymphoma was detected during the follow-up after the diagnosis of LCV. Cutaneous LCV was found in all of the cases in which a malignancy was detected.

When the laboratory examinations of the patients were assessed, the most frequently found abnormal laboratory parameter was an elevated erythrocyte sedimentation rate (ESR), detected in 49 (65.3\%) patients, followed by high C-reactive protein (CRP) ( $n=35,46.7 \%)$, and leukocytosis ( $n=16,21.3 \%$ ).

Joint involvement was found in 20 (26.7\%) patients, as arthritis and/or arthralgia, GIS involvement in 14 (18.6\%), as abdominal pain and/or occult blood positivity, and renal involvement in 20 (26.7\%), as haematuria and/or proteinuria. Systemic involvement was compared with the prevalence and type of lesions. No significant relationship was found between the presence of systemic involvement and the type or prevalence of the lesions $(p>0.05)$.

When the patients were assessed in terms of their treatment options, topical and symptomatic treatments, such as bed rest, topical corticosteroids, and/or systemic antihistamines, were used in 46 (61.3\%) patients. Twelve (16\%) patients received systemic steroids, 9 (12\%) received colchicine, 7 (9.3\%) received dapsone, and 1 (1.3\%) received azathioprine therapy (Table 1 ).

Of the 75 patients, 24 (32\%) were diagnosed with HSP by means of clinicopathological correlations; of those patients, 15 (62.5\%) were males, and 9 (37.5\%) were females. The male/female ratio was thus 1.67 . While the mean age of the patients diagnosed with HSP was 26.83 years, the mean age of the patients without HSP was 52.45 years. There was a statistically significant difference between the two groups ( $p<0.05)$. As for the seasonal distribution of the patients with HSP, 4 (23.5\%) patients presented in the spring, $7(28 \%)$ in the summer, $1(10 \%)$ in the autumn, and 12 (52.2\%) in the winter.

The systemic involvement locations and frequency of the patients with HSP were determined. While haematuria and proteinuria were seen together in 11 of 20 (83.3\%) patients with renal involvement, of the remaining $9 \mathrm{pa}$ tients, only proteinuria was found in $4(20 \%)$ and only haematuria in 5 (25\%). Of the 17 (70.8\%) patients with joint involvement, 6 had arthritis. While GIS involvement was detected in 14 (58.3\%) patients, occult blood in stool was positive in 5 (20.8\%) (Table 2).

High leukocyte and CRP levels and an elevated ESR at onset were assessed according to the presence of renal, joint and gastrointestinal involvement. No statistically significant difference was found $(p>0.05)$. 
Table 1. Treatment options in patients with vasculitides

\begin{tabular}{lll}
\hline Treatment options & $\boldsymbol{N}$ & $\%$ \\
\hline Symptomatic treatments & 46 & 61.3 \\
\hline Systemic steroid & 12 & 16 \\
\hline Colchicines & 9 & 12 \\
\hline Dapsone & 7 & 9.3 \\
\hline Azathioprine & 1 & 1.3 \\
\hline
\end{tabular}

Patients diagnosed with HSP were divided into two groups: those under 16 and those over 16 years of age. The presence of renal involvement, joint involvement, and GIS involvement was assessed in the groups; no statistically significant difference was found between them $(p>0.05)$.

\section{Discussion}

A wide variety of vasculitis conditions exists, and these are grouped according to the size of the affected vessels and the dominant clinical pattern. A new nomenclature for the various forms of systemic vasculitis was published in January 2013 [6], which is an update of the nomenclature system published in 1994 by the first International CHCC on the Nomenclature of Systemic Vasculitides.

Patients diagnosed with LCV were classified according to the 2012 Revised International CHCC Nomenclature of Vasculitides. In this classification, cutaneous leukocytoclastic vasculitis is a clinical term that generally refers to an immune complex-mediated small vessel vasculitis of the skin that spares internal organs and usually follows drug exposure or infection [6, 9-12]. In the study by Tai et al. [8] involving 93 adult patients, 68 (73.2\%) patients were classified as cutaneous LCV, 11 (11.8\%) as HSP, 5 (5.4\%) as microscopic polyangiitis, 2 (2.2\%) as Wegener's granulomatosis, 1 (1.1\%) as Churg-Strauss syndrome, $1(1.1 \%)$ as essential cryoglobulinemic vasculitis, and $4(4.3 \%)$ as septic vasculitis. In the study by Blanco et al. [13] involving 303 patients (172 adults, 131 children), HSP was found in 155 (51.6\%), cutaneous LCV in 84 (27.7\%), essential mixed cryoglobulinemia in 11 (3.6\%), polyarteritis nodosa (PAN) in 17 (5.6\%), Wegener's granulomatosis in $4(1.3 \%)$, and Churg-Strauss syndrome in $2(0.6 \%)$ patients. In the study by Jokar and Mirfeizi [9] involving 721 patients, the frequency distribution of vasculitic disorders was Behçet's disease $63.6 \%$, Wegener's granulomatosis 6.8\%, Takayasu's arteritis 6\%, Churg-Strauss syndrome $1.8 \%$, cryoglobulinemic vasculitis $0.3 \%$, and HSP $3.5 \%$. Of the 75 patients (62 adults, 13 children) involved in our study, 50 (66.6\%) were classified as cutaneous LCV, 24 (32\%) as HSP, and 1 (1.3\%) as cryoglobulinemic vasculitis. We believe that the differences in the classifi-
Table 2. Systemic involvement in patients with HenochSchönlein purpura

\begin{tabular}{lcc}
\hline Systemic involvement & $\boldsymbol{N}$ & $\%$ \\
\hline Joint involvement & 17 & 70.8 \\
\hline Arthralgia & 17 & 70.8 \\
\hline Arthritis & 6 & 25 \\
\hline GIS involvement & 14 & 58.3 \\
\hline Stomach ache & 14 & 58.3 \\
\hline FOB positive & 5 & 20.8 \\
\hline Renal involvement & 20 & 83.3 \\
\hline Haematuria & 5 & 25 \\
\hline Proteinuria & 4 & 20 \\
\hline Haematuria + proteinuria & 11 & 55 \\
\hline
\end{tabular}

cation distribution among these studies are associated with the numbers of the patients involved and the adult/ child patient ratios.

While almost half of the cases with cutaneous LCV are idiopathic, the other half are associated with medications, infections, lymphoproliferative disorders, solid tumours, connective tissue diseases, and inflammatory diseases [2-5]. The most frequent aetiological cause was medication usage in the study by Martinez-Taboada et al. [14], idiopathic in the study by Tai et al. [8], collagen tissue diseases in Blanco et al. [13], medication usage in Sahin et al. [15], streptococcal infections in Jessop [16], infections in Gyselbrecht et al. [17], and connective tissue diseases in Ekenstam et al. [18]. In our study, idiopathic cases ranked at the top, at $40 \%$, consistent with literature reports, and infections were second, at 26\%.

Therapeutic and diagnostic medications account for approximately $10 \%$ of vasculitic skin lesions. Vasculitis triggered by medications typically begins within 7-21 days after the start of the medication. Medications leading to leukocytoclastic vasculitis include $\beta$-lactam antibiotics, NSAIDs, sulphonamides, quinolones, and diuretics. In the study by Tai et al. [8], cephalosporin was found to account for the aetiology in $3.2 \%$ of patients $(n=3)$, penicillin in $2.2 \%(n=2)$, vancomycin in $2.2 \%(n=2)$, quinolones in $1.1 \%(n=1)$, rifampicin in $1.1 \%(n=1)$, NSAIDs in $2.2 \%(n=2)$, and other medications in $5.4 \%(n=5)$. In the study by Sahin et al. [15], antibiotics $(n=5)$, NSAIDs $(n=4)$, docetaxel $(n=1)$, lisinopril $(n=1)$, phenytoin $(n=1)$, and the combination of telmisartan and couma$\operatorname{din}(n=1)$ were involved. In our study, antibiotics $(n=4)$, NSAIDs $(n=2)$, calcium channel blockers $(n=1)$, ACE inhibitors $(n=1)$, lamotrigine $(n=1)$, ursodeoxycholic acid $(n=1)$, and a combination of propylthiouracil and a $\beta$-blocker $(n=1)$ were the medications detected. 
Cutaneous vasculitis may be associated with malig nancies and may behave as a paraneoplastic syndrome. Generally, vasculitis has been found to be associated with haematological malignancies. It has been reported that vasculitis may appear either before, concurrently, or after the malignancy diagnosis $[19,20]$. The most frequently accompanying solid organ malignancies include lung cancer, prostate cancer, breast cancer, colon cancer, and kidney cancer[19-21]. The mechanisms underlying the development of paraneoplastic vasculitis remain unknown. Some have asserted that the association is completely incidental. The most popular theory is that the vasculitis develops due to damage by immunocomplexes that have developed against tumour antigens to the vessel endothelium [21, 22]. Additionally, it has been reported that malignancies increase blood viscosity and, as a result, increase the time for the immunocomplexes to damage the endothelium [22]. Some researchers have implicated proinflammatory cytokines produced by tumour cells in the pathogenesis of vasculitis [23].

In many studies, vasculitis accompanying a malignancy is associated with the agents used to treat the malignancy. Tamoxifen [24], anastrozole [25], etoposide [26], erlotinib [27], and capecitabine [28] are agents that have been implicated in the literature. Again, subclinical infections suffered by these patients based on immunosuppression may also cause vasculitis [29, 30]. Fain et al. [20] examined 60 patients diagnosed with vasculitis accompanying a malignancy and found 24 solid organ malignancies in 22 (36.7\%) patients and haematological malignancies in 38 (63.3\%) patients. In the study by Fain et al. [20], systemic vasculitides accompanying solid organ malignancies were found to be PAN $(n=7)$, Wegener's granulomatosis $(n=2)$, and microscopic polyangiitis $(n=1)$. Solans-Laque et al. [19] reported 15 cases with vasculitis accompanying solid tumours in their study. While the most frequently accompanying vasculitis was again cutaneous LCV $(n=9)$, others were HSP $(n=2)$, PAN $(n=1)$, and giant cell arteritis $(n=3)$. In our study, malignancy was found in four patients. Of these, rheumatoid arthritis in one patient and the use of tamoxifen and a urinary tract infection in 1 patient accompanied the malignancy. Three of the patients were diagnosed with solid organ malignancies. While breast carcinoma, Kaposi's sarcoma, and malignant melanoma were the accompanying solid organ malignancies, non-Hodgkin lymphoma $(n=1)$ was the accompanying haematological malignancy. Cutaneous LCV was monitored in all of the patients in compliance with the literature. While 3 patients upon their diagnosis of vasculitis had previously diagnosed malignancies, malignancy was found in 1 patient after the diagnosis of vasculitis, during followup visits.

For LCV, the most frequently seen clinical finding is palpable purpura [31]. In our study, we also found palpable purpura in $65 \%$ of the patients, consistent with the literature. Koutkia et al. [31] stated that the presence of vesiculobullous type lesions was indicative of a more widespread and severe form of the disease. In our study, however, we did not see any statistically significant differences between the type or prevalence of the lesion, severity of the disease, or the presence of systemic involvement $(p>0.05)$.

The LCV lesions are generally asymptomatic. The presence of complaints such as fever, arthralgia, myalgia, headache, and abdominal pain indicates systemic disease as well [12]. In the study of Sais et al. [32], a rash was found in $41.4 \%$ of the patients and pain in $30 \%$. Arthralgia was the most frequently seen non-cutaneous finding, in $36.7 \%$ of patients. However, in the study of Sahin et al. [15], 45\% of patients were asymptomatic, while again arthralgia (33.4\%) was the most frequent non-cutaneous finding. In our study, while $64 \%$ of the patients were asymptomatic $(n=48)$, the most frequently accompanying non-cutaneous finding was joint pain, in $26.7 \%$ of the patients $(n=20)$, as in the studies of Sais et al. [32] and Sahin et al. [15].

For patients diagnosed with LCV, determining the aetiology and systemic involvement is important. In the reported studies, the ESR accompanies LCV in more than $50 \%$ of patients and may indicate a systemic disease. In the study by Sais et al. [32], leukocytosis, the ESR, and thrombocytosis were found, consistent with systemic involvement. However, in the studies of Sahin et al. [15] and Tai et al. [8], while the ESR was the most frequently seen pathological laboratory finding, there was no relationship between the high sedimentation and systemic involvement. In our study, the most frequently seen pathological laboratory finding was also the ESR, found in $65.3 \%$ $(n=49)$. However, we also found no link between the ESR, leukocytosis, or high CRP and systemic involvement.

The HSP is defined as a tetrad consisting of palpable purpura, arthritis, GIS involvement, and nephritis [33, 34]. It is seen more frequently in childhood [34] and is 1.5-fold more frequent in male than female children [13]. While the mean age of the patients diagnosed with HSP in our study was 26.83 years, the mean age of the patients diagnosed with cutaneous LCV was 51.45 years, and this was a significant difference $(p<0.05)$. The male/female ratio was 1.67 , consistent with the literature. However, in the studies carried out by Garcia-Porrua et al. [35] and Calvino et al. [36], it was seen more frequently in females. The HSP shows a seasonal pattern, with the greatest frequency in winter [37]. In the study by Saulsbury [38], it was reported that $2 / 3$ of the cases were seen in the autumn or winter. In the study by Akgün et al. [39], cases were seen most frequently in autumn (29.7\%), followed by winter $(25.1 \%)$. In our study, cases were seen most frequently in winter (52.2\%), consistent with the literature. The reason may depend on infectious triggers that play a role in HSP pathogenesis. 
The HSP is a vasculitis progressing with systemic involvement. In studies, various ratios have been reported with respect to systemic involvement. Farahnak [40] found $73.3 \%$ joint involvement and $68.6 \%$ GIS involvement in their study of 45 patients. They classified renal involvement and found haematuria in $88.6 \%$ of patients, haematuria and proteinuria in $66.7 \%$, nephrotic syndrome in $17.8 \%$, acute nephritic syndrome in $8.9 \%$, and nephritic-nephrotic syndrome in $13.3 \%$ of patients. Fretzayas et al. [41] also reported $91.8 \%$ joint involvement, 40.5\% GIS involvement, and $25.7 \%$ renal involvement. Peru et al. [42] reported 66\% joint involvement, 56\% GIS involvement, and 30\% renal involvement. Akgün et al. [39] also found $67.5 \%$ joint involvement, $46.7 \%$ GIS involvement, $22.6 \%$ renal involvement, and $0.9 \%$ testicular involvement in their study. Saulsbury [38] reported 50-80\% renal involvement in adults and $20-40 \%$ in children. In our study, we found joint involvement in 17 (70.8\%) of the patients, as arthritis and/or arthralgia, GIS involvement in 14 (58.3\%), as abdominal pain and/ or occult blood positivity in stool, and renal involvement in 20 (83.4\%) patients, as haematuria and/or proteinuria.

In various studies, it has been reported that the older the patient is, the higher the rate of renal involvement in HSP. Farahnak et al. [40] found higher renal involvement in HSP in adults and older children. Similarly, Kaku et al. [43] reported that renal involvement frequency increased with age. While Akgün et al. [39] did not find a relationship among joint involvement, GIS involvement, central nervous system involvement, and scrotal involvement, they found a significant relationship with renal involvement. In our study, however, no significant relationship was found between renal involvement, GIS involvement, or joint involvement and age $(p>0.005)$.

\section{Conclusions}

Our study confirms that LCV occurs around the age of 40 and presents with palpable purpura on the lower extremities. The LCV is more common in females. The disease is frequently idiopathic and usually progresses asymptomatically. Drugs, most frequently antibiotics, are involved in the aetiology of LCV. An elevated ESR is the most common laboratory finding and is also associated with connective tissue diseases and malignancy. The LCV is usually self-limited. In comparison, HSP occurs at a younger age, is more common in males, and is most frequent in winter. There is no relationship between renal involvement and age in HSP.

\section{Conflict of interest}

The authors declare no conflict of interest.

\section{References}

1. Ratzinger G, Zelger BG, Carlson JA, et al. Vasculitic wheel - an algorithmic approach to cutaneous vasculitis. J Dtsch Dermatol Ges 2015; 13: 1092-117.

2. Chung L, Kea B, Fiorentino DF. Cutaneous vasculitis. In: Dermatology. $2^{\text {nd }}$ ed. Bolognia JL, Jorizzo JL, Rapini RP (eds). Elsevier Limited, Spain 2008; 347-67.

3. Soter NA, Diaz-perez JL. Cutaneous necrotizing venulitis. In: Fitzpatrick's Dermatology in General Medicine. $7^{\text {th }}$ ed. Wolff K, Goldsmith LA, Katz SI, et al. (eds). McGraw-Hill Companies, New York 2008; 1599-606.

4. Khasnis A, Langford CA. Update on vasculitis. J Allergy Clin Immunol 2009; 123: 1226-36.

5. Hussain N, Mustafa U, Davis J, et al. Indomethacin-related leukocytoclastic vasculitis: a case report and review of literature. Case Rep Dermatol 2013; 5: 33-7.

6. Jennette JC, Falk RJ, Bacon PA, et al. 2012 Revised International Chapel Consensus Conference Nomenclature of Vasculitides. Arthritis Rheum 2013; 65: 1-11.

7. Sunderkötter C, Pappelbaum KI, Ehrchen J. Cutaneous symptoms of various vasculitides. Hautarzt 2015; 66: 589-98.

8. Tai YJ, Chong AH, Williams RA, et al. Retrospective analysis of adult patients with cutaneous leukocytoclastic vasculitis. Australas J Dermatol 2006; 47: 92-6.

9. Jokar M, Mirfeizi Z. Epidemiology of vasculitides in Khorasan Province, Iran. Iran J Med Sci 2015; 40: 362-6.

10. Chen KR, Carlson JA. Clinical approach to cutaneous vasculitis. Am J Clin Dermatol 2008; 9: 71-92.

11. Jennette JC, Falk RJ. Necrotizing arteritis and small vessel vasculitis. In: The Autoimmune Diseases. Rose N, Mackay I (eds). Academic Press 2006; 899-920.

12. Jennette JC, Falk RJ. Small vessel vasculitis. N Engl J Med 1997; 337: 1512-23.

13. Blanco R, Martinez-Taboada VM, Rodriguez-Valverde V, et al. Cutaneous vasculitis in children and adults: associated diseases and etiologic factors in 303 patients. Medicine (Baltimore) 1998; 77: 403-18.

14. Martinez-Taboada VM, Blanco R, Garcia-Fuentes M, et al. Clinical features and outcome of 95 patients with hypersensitivity vasculitis. Am J Med 1997; 102: 186-91.

15. Sahin EB, Hapa A, Elçin G, et al. Leukocytoclastic vasculitis retrospective analysis of 60 patients. Turk Dermatoloji Dergisi 2011; 5: 85-91.

16. Jessop SJ. Cutaneous leukocytoclastic vasculitis: a clinical and aetiological study. Br J Rheumatol 1995; 34: 942-5.

17. Gyselbrecht L, De Keyser F, Ongenae K, et al. Etiological factors and underlying conditions in patients with leukocytoclastic vasculitis. Clin Exp Rheumatol 1996; 14: 665-8.

18. Ekenstam EA, Callen JP. Cutaneous leukocytoclastic vasculitis. Clinical and laboratory features of 82 patients seen in private practice. Arch Dermatol 1984; 120: 484-9.

19. Solans-Laque R, Bosch-Gil JA, Perez-Bocanegra C, et al. Paraneoplastic vasculitis in patients with solid tumors: report of 15 cases. J Rheumatol 2008; 35: 294-304.

20. Fain O, Hamidou M, Cacoub P, et al. Vasculitides associated with malignancies: analysis of sixty patients. Arthritis Rheum 2007; 57: 1473-80.

21. Fortin PR. Vasculitides associated with malignancy. Curr Opin Rheumatol 1996; 8: 30-3.

22. Wooten MD, Jasin HE. Vasculitis and lymphoproliferative diseases. Semin Arthritis Rheum 1996; 26: 564-74.

23. Tatsis E, Reinhold-Keller E, Steindorf K, et al. Wegener's granulomatosis associated with renal cell carcinoma. Arthritis Rheum 1999; 42: 751-6. 
24. Ruiz Villaverde R, Blasco Melguizo J, Martin Sanchez MC, et al. Annular leukocytoclastic vasculitis: response to dapsone. J Eur Acad Dermatol Venereol 2002; 16: 544-6.

25. Fredenberg MD, Malkinson FD. Sulfone therapy in the treatment of leukocytoclastic vasculitis. Report of three cases. Dermatology 1987; 16: 772-8.

26. Fortson JS, Zone JJ, Hammond ME. Hypocomplementemic urticarial vasculitis syndrome responsive to dapsone. J Am Acad Dermatol 1986; 15: 1137-42.

27. Callen JP, Spencer LV, Burruss JB, et al. Azathioprine. An effective, corticosteroid sparing therapy for patients with recalcitrant cutaneous lupus erythematosus or with recalcitrant cutaneous leukocytoclastic vasculitis. Arch Dermatol 1991; 127: 515-22.

28. Al Shamsi HO, Kee BK, Tetzlaff MT, et al. Capecitabine-induced leukocytoclastic vasculitis under neoadjuvant chemotherapy for locally advanced colorectal cancer. J Gastrointest Oncol 2015; 6: E40-3.

29. Loricera J, Calvo-Rio V, Sanjuan FO, et al. The spectrum of paraneoplastic cutaneous vasculitis in a defined population: incidence and clinical features. Medicine 2013; 92: 331-43.

30. Greer JM, Longley S, Edwards NL, et al. Vasculitis associated with malignancy: experience with 13 patients and literature review. Medicine 1988; 67: 220-30.

31. Koutkia P, Mylonakis E, Rounds S, et al. Leukocytoclastic vasculitis: an update for the clinician. Scand I Rheumatol 2001; 30: 315-22.

32. Sais G, Vidaller A, Jungle A, et al. Prognostic factors in leukocytoklastic vasculitis. A clinicopathologic study of $160 \mathrm{pa}-$ tients. Arch Dermatol 1998; 134: 309-15.

33. Fiorentino DF. Cutaneous vasculitis. J Am Acad Dermatol 2003; 48: 311-40.

34. Nong BO, Huang YI, Chuang CM, et al. Fifteen-year experience of children with Henoch-Schönlein purpura in southern Taiwan, 1991-2005. J Microbiol Immunol Infect 2007; 40: 371-6.

35. Garcia-Porrua C, Calvino MC, Llorca J, et al. Henoch Schönlein purpura in children and adults: clinical differences in a defined population. Semin Arthritis Rheum 2002; 32: 149-56.

36. Calvino MC, Llorca J, Garcia-Porrua C, et al. Henoch Schönlein purpura in children from northwestern Spain: a 20-year epidemiologic and clinical study. Medicine (Baltimore) 2001; 80: 279-90.

37. Miller ML, Pachman ML. Vasculitis syndromes. In: Nelson Textbook of Paediatrics. Kliegman RM, Behrman RE, Jenson HB, Stanton BF (eds). WB Saunders Co, Philadelphia 2007; 1042-9.

38. Saulsbury FT. Henoch Schönlein purpura in children: report of 100 patients and review of the literature. Medicine 1999; 78: 395-409.

39. Akgün C, Akbayram S, Kaya A, et al. The clinical analysis of childhood Henoch-Schönlein purpura patients. Turkderm 2012; 46: 73-7.

40. Farahnak A. Childhood Henoch-Schönlein nephritis: a multivariate analysis of clinical features and renal morphology at disease onset. Iran J Kidney Dis 2009; 3: 17-21.

41. Fretzayas A, Sionti I, Moustaki M, et al. Henoch-Schönlein purpura: a long term prospective study in Greek children. J Clin Rheumatol 2008; 14: 324-31.

42. Peru H, Soylemezoğlu O, Bakkaloğlu SA, et al. HenochSchönlein purpura in childhood: clinical analysis of 254 cases over a 3 year period. Clin Rheumatol 2008; 27: 1087-92.
43. Kaku Y, Nohara K, Honda S. Renal involvement in HenochSchönlein purpura: a multivariate analysis of prognostic factors. Kidney Int 1998; 53: 1755-9. 